\title{
Estatísticas de Segunda Ordem para Canais de Desvanecimento $\eta$ - $\mu$ : Teoria e Aplicações
}

\author{
Daniel Benevides da Costa, Student Member, IEEE, Michel Daoud Yacoub e José Cândido Silveira Santos Filho
}

\begin{abstract}
Resumo-Este artigo apresenta expressões, em termos de uma única integral, para a taxa de cruzamento de nível e duração média de desvanecimento de canais $\eta$ - $\mu$. As expressões são validadas reduzindo-as a alguns casos particulares (Nakagami-m, Hoyt e Rayleigh) e através da simulação Monte Carlo. Além disso, baseando-se nas novas estatísticas encontradas, formulações exatas e aproximações precisas, em função de uma única integral, para as estatísticas de cruzamento de combinadores multirramos por ganho igual e razão máxima, respectivamente, operando em canais independentes de desvanecimento Hoyt são apresentadas. As soluções exatas correspondentes disponíveis na literatura são dadas em termos de múltiplas integrais. Um número significativo de expressões em forma fechada para as estatísticas conjuntas da envoltória, fase e suas derivadas temporais podem ser obtidas a partir de nossas formulações. Para finalizar, a taxa de cruzamento de fase para canais de desvanecimento $\eta$ - $\mu$ é calculada e validada.
\end{abstract}

Palavras-Chave-Métodos de aproximação, duração média de desvanecimento, canais de desvanecimento generalizados, taxa de cruzamento de nível, taxa de cruzamento de fase.

Abstract - In this paper, we derive the level crossing rate and average fade duration of $\eta-\mu$ fading channels in a singlefold integral form. The expressions are thoroughly validated by reducing them to some particular known cases (Nakagami-m, Hoyt, and Rayleigh) and, more generally, by means of Monte Carlo simulation. Furthermore, based on the new results, we provide alternative singlefold integral exact formulations and highly-accurate approximations to the level-crossing statistics of multibranch maximal-ratio and equal-gain combining systems, respectively, operating over independent Hoyt fading channels. The corresponding exact solutions currently available in the literature are written in a multifold integral form. A significant number of new closed-form expressions envolving the joint statistics of the envelope, phase, and their time derivatives shall be obtained from our formulations. In passing, the phase crossing rate for $\eta-\mu$ fading channels is also attained and thoroughly validated.

Keywords-Approximation methods, average fade duration, generalized fading channels, level crossing rate, phase crossing rate.

\section{INTRODUÇÃO}

Em comunicações sem fio, uma modelagem precisa do canal de propagação é importante. Dessa forma, ao longo dos anos, um grande número de modelos de canais que seguem o comportamento do sinal rádio-móvel tem sido propostos. A variação do sinal à curto prazo (desvanecimento rápido) é descrita por algumas distribuições, tais como Rayleigh,

Daniel Benevides da Costa, Michel Daoud Yacoub e José Cândido Silveira Santos Filho, Departamento de Comunicações, Faculdade de Engenharia Elétrica e Computação, Universidade Estadual de Campinas, Campinas, Brasil, E-mails: daniel@wisstek.org, michel@wisstek.org, candido@wisstek.org. Este trabalho foi parcialmente financiado pela FAPESP (05/59259-7).
Hoyt (Nakagami-q), Rice, Nakagami-m e Weibull. Dentre os cenários de desvanecimento à curto prazo, Nakagami- $m$ tem recebido uma atenção especial devido à sua facilidade de manipulação e grande aplicabilidade. Por outro lado, em algumas situações, a cauda de sua distribuição não segue precisamente as estatísticas reais. Sendo assim, com o intuito de tornar os modelos existentes mais flexíveis, vários estudos têm sido realizados na literatura [1], [2].

Recentemente [3], [4], o modelo de desvanecimento $\eta$ - $\mu$, que inclui os modelos de Hoyt e Nakagami- $m$ como casos especiais, tem sido investigado. Sua flexibilidade o torna mais adaptável a situações no qual nenhuma destas duas distribuições (Hoyt e Nakagami-m) produzem um bom ajuste, principalmente na parte da cauda, onde tal probabilidade é maior. Além disso, sabe-se que a distribuição $\eta$ - $\mu$ pode ser usada para aproximar satisfatoriamente a distribuição da soma de variáveis Hoyt independentes e não-idênticas, tendo potências médias e graus de desvanecimento arbitrários [5]. O ganho de flexibilidade da distribuição $\eta-\mu$ é obtido com a inclusão de um novo parâmetro. Em relação a Nakagami- $m$, o parâmetro adicional é $\eta$, no qual este considera o desbalanceamento de potência das componentes em fase e em quadratura do sinal desvanecido ou, equivalentemente, a correlação entre estas componentes. Já em relação a distribuição de Hoyt, o parâmetro adicional é $\mu$, que leva em conta a clusterização dos sinais de multipercurso. Esta nova proposta de modelagem vem com uma tentativa de melhor descrever o sinal desvanecido em um ambiente no qual os campos de espalhamento difusos resultam a partir de superfícies correlacionadas, caracterizando um ambiente real não-homogêneo [6].

O objetivo deste artigo é explorar as estatísticas de segunda ordem de canais de desvanecimento $\eta-\mu$. Logo, expressões originais, exatas e em forma fechada referentes as estatísticas $\eta$ - $\mu$ são apresentadas, nas quais estas incluem Hoyt e Nakagami- $m$ como casos particulares. As expressões exatas obtidas para a taxa de cruzamento de nível (LCR) e duração média de desvanecimento (AFD) são usadas para aproximar a LCR e AFD de $M$ canais Hoyt usando combinação por ganho (EGC), onde integrais múltiplas e complexas fornecem a solução exata [7, Eq. 14]. Além disso, estas mesmas expressões exatas constituem soluções exatas para a LCR e AFD de $M$ canais Hoyt usando combinação por razão máxima (MRC), substituindo assim as integrais múltiplas e complexas que representam a solução exata original [7, Eq. 18]. Os resultados analíticos são completamente validados reduzindo as expressões para alguns casos particulares e por meio de simulação. 


\section{O Modelo De Desvanecimento $\eta-\mu$}

Nesta seção, o modelo de desvanecimento $\eta$ - $\mu$ proposto em [3], [4] será revisto. Tal modelo considera o sinal composto por clusters de ondas de multipercurso, tal que dentro de qualquer um dos clusters as fases das ondas espalhadas são aleatórias e têm atrasos temporais similares, com o espalhamento do atraso dos diferentes clusters sendo relativamente grande. A envoltória $R$ do sinal desvanecido $\eta$ - $\mu$ pode ser escrita como

$$
R^{2}=\sum_{i=1}^{2 \mu}\left(X_{i}^{2}+Y_{i}^{2}\right)
$$

onde $X_{i}$ e $Y_{i}$ são processos gaussianos em fase e em quadratura de médias nula, isto é, $E\left(X_{i}\right)=E\left(Y_{i}\right)=0$, e $E(\cdot)$ denota o operador esperança. A distribuição $\eta-\mu$, como seu próprio nome já diz, é escrita em termos de dois parâmetros físicos, $\eta$ e $\mu$, e pode ser encontrada em dois formatos distintos, Formato 1 e Formato 2, correspondendo assim a dois modelos físicos diferentes [4]. Superficialmente falando, o parâmetro $\mu$ está relacionado ao número de clusters de ondas de multipercurso no ambiente, enquanto que o parâmetro $\eta$ está relacionado à razão das potências (no Formato 1) ou correlação (no Formato 2) entre as ondas de multipercurso das componentes em fase e em quadratura. Tais conceitos serão detalhados na próxima subseção. A função densidade de probabilidade (PDF) da envoltória $\eta$ - $\mu$ é dada por [3], [4]

$$
\begin{aligned}
f_{R}(r)=\frac{4 \sqrt{\pi} \mu^{\mu+\frac{1}{2}} h^{\mu}}{\Gamma(\mu) H^{\mu-\frac{1}{2}} \hat{r}}\left(\frac{r}{\hat{r}}\right)^{2 \mu} & \exp \left[-2 \mu h\left(\frac{r}{\hat{r}}\right)^{2}\right] \\
& \times I_{\mu-\frac{1}{2}}\left[2 \mu H\left(\frac{r}{\hat{r}}\right)^{2}\right],
\end{aligned}
$$

onde $\hat{r}=\sqrt{\Omega}=\sqrt{E\left(R^{2}\right)}, \Gamma(z)=\int_{0}^{\infty} t^{z-1} \exp (-t) d t$ é a função Gama, $I_{\nu}[\cdot]$ é a função de Bessel modificada de primeiro tipo e ordem arbitrária $\nu$ [8, Eq. 9.6.20], e $\mu>0$ está relacionado ao número de clusters de multipercurso tal que

$$
\mu=\frac{E^{2}\left(R^{2}\right)}{2 V\left(R^{2}\right)} \times\left[1+\left(\frac{H}{h}\right)^{2}\right]
$$

Os parâmetros $h$ e $H$ são funções de $\eta$ e serão definidos em seguida para os dois formatos distintos. A partir de (2), o $k$ ésimo momento de $R$ é calculado como

$$
\begin{aligned}
E\left(R^{k}\right) & =\frac{\Gamma\left(2 \mu+\frac{k}{2}\right) \Omega^{\frac{k}{2}}}{h^{\mu+\frac{k}{2}}(2 \mu)^{\frac{k}{2}} \Gamma(2 \mu)} \\
& \times{ }_{2} F_{1}\left(\mu+\frac{k}{4}+\frac{1}{2}, \mu+\frac{k}{4} ; \mu+\frac{1}{2} ;\left(\frac{H}{h}\right)^{2}\right),
\end{aligned}
$$

onde ${ }_{2} F_{1}(\cdot, \cdot ; \cdot ; \cdot)$ é a função hipergeométrica [8, Eq. 15.1.1]. A função de distribuição cumulativa (CDF) da envoltória $\eta-\mu$ pode ser expressa como

$$
F_{R}(r)=1-Y_{\mu}\left(\frac{H}{h}, \sqrt{2 h \mu} \frac{r}{\hat{r}}\right),
$$

onde [4]

$$
\begin{aligned}
Y_{v}(\lambda, \beta) \triangleq & \frac{2^{-v+\frac{3}{2}} \sqrt{\pi}}{\Gamma(v)} \frac{\left(1-\lambda^{2}\right)^{v}}{\lambda^{v-\frac{1}{2}}} \\
& \int_{\beta}^{\infty} x^{2 v} \exp \left(-x^{2}\right) I_{v-\frac{1}{2}}\left[\lambda x^{2}\right] d x .
\end{aligned}
$$

As estatísticas aqui obtidas serão detalhadas apenas para o Formato 1. Porém, como será mostrado na Subseção II-C, um formato pode ser convertido no outro por meio de uma simples transformação bilinear.

\section{A. O Modelo de Desvanecimento $\eta-\mu$ - Formato 1}

O modelo de desvanecimento para a distribuição $\eta$ - $\mu$ (Formato 1) considera um sinal composto por clusters de ondas de multipercurso propagando-se em um ambiente nãohomogêneo. As componentes em fase e em quadratura do sinal desvanecido dentro de cada cluster são mutuamente independentes e possuem potências distintas.

Neste formato, $0<\eta<\infty$ representa a razão entre as potências das componentes em fase e em quadratura de cada cluster de multipercurso, isto é, $\eta=E\left(X_{i}^{2}\right) / E\left(Y_{i}^{2}\right)$. Além disso, temos que $h=\left(2+\eta^{-1}+\eta\right) / 4$ e $H=\left(\eta^{-1}-\eta\right) / 4$, resultando $H / h=(1-\eta) /(1+\eta)$. Perceba que para $0<\eta \leq 1$ a distribuição da envoltória assume os mesmos valores para $0<\eta^{-1} \leq 1$, fazendo com que a envoltória seja simétrica em $\eta=1$.

\section{B. O Modelo de Desvanecimento $\eta-\mu$ - Formato 2}

O modelo de desvanecimento para a distribuição $\eta-\mu$ (Formato 2) considera um sinal composto por clusters de ondas de multipercurso propagando-se em um ambiente nãohomogêneo. As componentes em fase e em quadratura do sinal desvanecido dentro de cada cluster possuem as mesmas potências e são correlacionadas.

Neste formato, $-1<\eta<1$ é o coeficiente de correlação entre as componentes em fase e em quadratura de cada cluster de multipercurso, isto é, $\eta=E\left(X_{i} Y_{i}\right) / E\left(X_{i}^{2}\right)$ (ou, equivalentemente, $\left.\eta=E\left(X_{i} Y_{i}\right) / E\left(Y_{i}^{2}\right)\right)$. Além disso, segue que $h=1 /\left(1-\eta^{2}\right)$ e $H=\eta /\left(1-\eta^{2}\right)$, então $H / h=\eta$. Perceba que para $0<\eta \leq 1$ a distribuição da envoltória assume os mesmos valores para $-1<\eta \leq 0$, que implica na simetria da envoltória em torno de $\eta=0$.

\section{Formato 1 e Formato 2}

Da discussão acima, temos que $H / h=(1-\eta) /(1+\eta)$ para o Formato 1 e $H / h=\eta$ para o Formato 2 . Portanto, é fácil ver que um formato pode ser obtido a partir do outro pela relação bilinear $\eta_{1}=\left(1-\eta_{2}\right) /\left(1+\eta_{2}\right)$ ou, de forma equivalente, $\eta_{2}=\left(1-\eta_{1}\right) /\left(1+\eta_{1}\right)$, onde $\eta_{1}$ é o parâmetro $\eta$ no Formato 1 e $\eta_{2}$ é o parâmetro $\eta$ no Formato 2 .

\section{Casos Especiais}

Como já comentado antes, a distribuição $\eta$ - $\mu$ é uma distribuição de desvanecimento geral que inclui as distribuições de Hoyt (ou Nakagami- $q$ ) e Nakagami-m como casos especiais. 
Dessa forma, a distribuição de Hoyt (ou Nakagami-q) pode ser obtida a partir da distribuição $\eta$ - $\mu$ de forma exata fazendo $\mu=0.5$. Neste caso, o parâmetro de Hoyt (ou Nakagami-q) é dado por $b=-(1-\eta) /(1+\eta)\left(\right.$ ou $\left.q^{2}=\eta\right)$ no Formato 1 ou $b=-\eta$ (ou $q^{2}=(1-\eta) /(1+\eta)$ ) no Formato 2 . Já a distribuição de Nakagami- $m$ pode ser obtida a partir da distribuição $\eta-\mu$ de forma exata fazendo $\mu=m$ e $\eta \rightarrow 0$ ou $\eta \rightarrow \infty$ no Formato 1 , ou $\eta \rightarrow \pm 1$ no Formato 2 . De forma similar, ela pode ser também obtida para $\mu=m / 2$ e $\eta \rightarrow 1$ no Formato 1 or $\eta \rightarrow 0$ no Formato 2 .

\section{DistribuiçÃo CONJUNTA DA ENVOLTÓRIA E DA FASE}

Nesta seção, a PDF conjunta $f_{R, \dot{R}, \Theta} \dot{\Theta}(r, \dot{r}, \theta, \dot{\theta})$ da envoltória $R$, da fase $\Theta$, e de suas respectivas derivadas temporais $\dot{R}$ e $\dot{\Theta}$, é obtida. Como já mencionado, a análise será realizada apenas para o Formato 1. De [9], temos que as componentes em fase $X$ e em quadratura $Y$ do sinal em desvanecimento $\eta$ - $\mu$ são mutuamente independentes com PDFs marginais dadas por ${ }^{1}$

$$
f_{Z}(z)=\frac{\mu^{\mu}|z|^{2 \mu-1}}{\Omega_{Z}^{\mu} \Gamma(\mu)} \exp \left(-\frac{\mu z^{2}}{\Omega_{Z}}\right),-\infty<z<\infty,
$$

onde $Z \equiv X$ ou $Z \equiv Y$, como requerido. Além disso, $\Omega_{Z}=E\left(Z^{2}\right)$. Como $\eta=\Omega_{X} / \Omega_{Y}$, pode ser mostrado que $\Omega_{X}=\hat{r}^{2} \eta /(1+\eta)$ e $\Omega_{Y}=\hat{r}^{2} /(1+\eta)$ [9]. Cada componente $Z$ pode ser escrita como $Z=S|Z|$, onde $S=\operatorname{sgn}(Z)$ (sinal de $Z$ ) e $|Z|$ segue uma distribuição Nakagami-m [12]. Por conveniência, escreveremos $Z=S N$, no qual $N$ representa uma variável Nakagami- $m$. Diferenciando $Z$ em relação ao tempo, segue que $\dot{Z}=\dot{S} N+S \dot{N}$. Devido $S$ assumir valores constantes \pm 1 , exceto nos instantes de transição $(-1 \rightarrow+1 \mathrm{e}$ $+1 \rightarrow-1$ ), sua derivada temporal $\dot{S}$ é nula. Além disso, como $Z$ é contínuo, os instantes de transição ocorrem exatamente e somente nos instantes de cruzamento de zero de $Z$, implicando $N=|Z|$ nulo. Portanto, sempre $\dot{S} N=0$ e $\dot{Z}=S \dot{N}$. Em [13] foi mostrado que $\dot{N}$ é gaussiano e independente de $N$. Sabendo que $\dot{Z}=S \dot{N}$, então $\dot{Z}$ condicionado a $Z=S N$ segue também uma distribuição gaussiana, tendo os mesmo parâmetros da distribuição de $\dot{N}$. Portanto, $\dot{Z}$ é de fato independente de $Z$. De forma mais clara, $\dot{X}$ e $\dot{Y}$ seguem uma distribuição gaussiana de média nula com desvios padrões $\pi f_{m} \hat{r} \sqrt{\eta /(\mu(1+\eta))}$ e $\pi f_{m} \hat{r} \sqrt{1 /(\mu(1+\eta))}$, respectivamente, onde $f_{m}$ é o desvio Doppler máximo em Hz. Como já mencionado, $X$ é independente de $\dot{X}$ e $Y$ é independente de $\dot{Y}$. Sendo $X$ e $Y$ processos independentes, segue que $X, \dot{X}, Y$ e $\dot{Y}$ são mutuamente independentes. Finalmente, percebendo que $\dot{X}$ e $\dot{Y}$ seguem uma distribuição gaussiana com os parâmetros citados e que a PDF de $X$ e $Y$ é expressa em (7), a PDF conjunta $f_{X, \dot{X}, Y, \dot{Y}}(x, \dot{x}, y, \dot{y})$ é dada por $f_{X, \dot{X}, Y, \dot{Y}}(x, \dot{x}, y, \dot{y})=f_{X}(x) f_{\dot{X}}(\dot{x}) f_{Y}(y) f_{\dot{Y}}(\dot{y})$. Sabendo que $X$ e $Y$ podem ser escritos em termos da envoltória $R$ e fase $\Theta$ como $X=R \cos \Theta$ e $Y=R \sin \Theta$, segue que $\dot{X}=\dot{R} \cos \Theta-R \dot{\Theta} \sin \Theta$ e $\dot{Y}=\dot{R} \sin \Theta+R \dot{\Theta} \cos \Theta$.

\footnotetext{
${ }^{1}$ As componentes em fase $X$ e em quadratura $Y$ do sinal em desvanecimento $\eta$ - $\mu$ podem ser expressas em termos das componentes em fase $X_{i}$ e em quadratura $Y_{i}$ de cada um dos clusters de multipercurso que compõem o sinal como $X^{2}=\sum_{i=1}^{2 \mu} X_{i}^{2}$ e $Y^{2}=\sum_{i=1}^{2 \mu} Y_{i}^{2}$.
}

Seguindo o procedimento estatístico padrão de transformação de variáveis e após manipulações algébricas, a PDF conjunta $f_{R, \dot{R}, \Theta, \dot{\Theta}}(r, \dot{r}, \theta, \dot{\theta})$ é obtida como

$$
\begin{aligned}
& f_{R, \dot{R}, \Theta, \dot{\Theta}}(r, \dot{r}, \theta, \dot{\theta})=\frac{[(1+\eta) \mu]^{2 \mu+1}|\sin (2 \theta)|^{2 \mu-1} r^{4 \mu}}{2^{2 \mu} \pi^{3} f_{m}^{2} \eta^{\mu+\frac{1}{2}} \Gamma^{2}(\mu) \hat{r}^{4 \mu+2}} \\
& \times \exp \left\{-\frac{(1+\eta) \mu}{2 \hat{r}^{2}}\left[\frac{[\dot{r} \cos (\theta)-r \dot{\theta} \sin (\theta)]^{2}}{f_{m}^{2} \pi^{2} \eta}+\right.\right. \\
& \left.\left.+\frac{[\dot{r} \sin (\theta)+r \dot{\theta} \cos (\theta)]^{2}}{f_{m}^{2} \pi^{2}}+2 r^{2}\left(\frac{\cos ^{2}(\theta)}{\eta}+\sin ^{2}(\theta)\right)\right]\right\} .
\end{aligned}
$$

Para as condições apropriadas, como detalhadas antes, (8) reduz de maneira exata aos casos Nakagami- $m$ [10, Eq. 4] e Hoyt [11, Eq. 10]. Ainda a partir de (8), realizando as devidas integrações, algumas PDFs conjuntas exatas e em forma fechadas podem ser encontradas. Devido à limitação de espaço, elas não serão mostradas aqui.

\section{Taxas de Cruzamento e Duração Média de DESVANECIMENTO}

Seja $P$ um processo, $\dot{P}$ sua derivada temporal e $p$ um nível específico. A taxa de cruzamento do processo $P, N_{P}(p)$, é definida como

$$
N_{P}(p)=\int_{0}^{\infty} \dot{p} f_{P, \dot{P}}(p, \dot{p}) d \dot{p}
$$

onde $f_{P, \dot{P}}(\cdot, \cdot)$ é a PDF conjunta de $P$ e $\dot{P}$. A AFD $T_{P}(p)$ é obtida como

$$
T_{P}(p)=\frac{F_{P}(p)}{N_{P}(p)}
$$

onde $F_{P}(p)$ é a CDF de $P$. Na seqüência, a LCR, AFD e taxa de cruzamento de fase (PCR) serão obtidas para canais de desvanecimento $\eta-\mu$.

\section{A. $L C R$ e $A F D$}

A LCR e AFD referem-se às estatísticas da envoltória. Neste caso, em (9) e em (10), $P \equiv R$ and $p \equiv r$. A PDF conjunta $f_{R, \dot{R}}(r, \dot{r})$ é obtida a partir de (8) após as devidas integrações. Segue então que

$$
\begin{aligned}
N_{R}(r) & =\frac{f_{m} \sqrt{\pi}[(1+\eta) \mu]^{2 \mu-\frac{1}{2}} r^{4 \mu-1}}{2^{2 \mu-2} \eta^{\mu} \Gamma^{2}(\mu) \hat{r}^{4 \mu-1}} \\
& \int_{0}^{\frac{\pi}{2}}[\sin (2 \theta)]^{2 \mu-1} \sqrt{1+\eta-(1-\eta) \cos (2 \theta)} \\
& \times \exp \left\{-\frac{\left[(1+\eta)^{2}+\left(1-\eta^{2}\right) \cos (2 \theta)\right] \mu r^{2}}{2 \eta \hat{r}^{2}}\right\} d \theta .
\end{aligned}
$$

A AFD é obtida a partir de (11) e (5). 


\section{B. $P C R$}

PCR refere-se à estatística da fase. Neste caso, em (9), $P \equiv$ $\Theta$ e $p \equiv \theta$. A PDF conjunta $f_{\Theta, \dot{\Theta}}(\theta, \dot{\theta})$ é obtida a partir de (8), após as requeridas integrações. Segue então que

$$
N_{\Theta}(\theta)=\frac{f_{m} \sqrt{\pi} \eta^{\mu-\frac{1}{2}} \Gamma\left(2 \mu-\frac{1}{2}\right)|\sin (2 \theta)|^{2 \mu-1}}{2^{\frac{3}{2}} \Gamma^{2}(\mu)[1+\eta+(1-\eta) \cos (2 \theta)]^{2 \mu-1}} .
$$

Para determinadas condições de desvanecimento, como descritas anteriormente, (11) e (12) reduzem-se exatamente e respectivamente a LCR e PCR do sinal Nakagami- $m$ apresentadas em [13, Eq. 17] e [10, Eq. 12]. De forma análoga, a LCR assim como a PCR de canais de desvanecimento Hoyt dadas em [11, Eq. 13] e [14], respectivamente, podem ser obtidas a partir de (11) e (12).

\section{AplicAÇÕES}

A LCR e AFD de sistemas multirramos EGC e MRC operando em canais independentes de desvanecimento Hoyt são conhecidas na literatura e apresentadas em termos de múltiplas integrais [7, Eqs. 14 and 18]. Nesta seção, serão usadas as expressões para a LCR e AFD, obtidas na Subseção IV-A, como soluções exatas, simples e alternativas para a LCR e AFD de sistemas multirramos MRC operando em canais Hoyt. Da mesma forma, elas serão usadas como aproximações altamente precisas para a LCR e AFD de sistemas EGC sobre o mesmo cenário de desvanecimento. Em cada caso, os parâmetros da distribuição $\eta-\mu(\Omega, \eta$ e $\mu)$ serão devidamente calculados em termos dos parâmetros de Hoyt de cada ramo. $\mathrm{O}$ procedimento para tal será detalhado em seguida. Esta mesma abordagem pode ser usada para o caso Nakagami- $m$, no qual a solução exata é dada em [15], [16]. Por conveniência, somente o caso Hoyt será explorado neste artigo.

\section{A. Combinação por Razão Máxima}

Em MRC, a envoltória na saída do combinador $\tilde{R}$ é dada por

$$
\tilde{R}^{2}=\sum_{i=1}^{M} R_{i}^{2}
$$

onde $R_{i}$ é a envoltória do $i$-ésimo ramo, $i=1, \ldots, M$, e segue a distribuição de Hoyt com potência média $\Omega_{i}=E\left(R_{i}^{2}\right)$ e parâmetro de desvanecimento $b_{i}$. De fato, cada envoltória Hoyt $R_{i}$ pode ser modelada como a soma de variáveis quadráticas gaussianas independentes e desbalanceadas, tal que (13) coincide com o modelo de envoltória $\eta$ - $\mu$ (1) (isto é, $\tilde{R} \equiv R$ ) tendo a distribuição $\eta-\mu$ os parâmetros

$$
\begin{aligned}
\Omega & =M \Omega_{i}, \\
\mu & =M / 2, \\
\eta & =\frac{1+b_{i}}{1-b_{i}} .
\end{aligned}
$$

Usando (14) em (11) e (5), obtém-se formulações alternativas exatas, expressas como uma única integral, para a LCR e AFD de sistemas MRC operando em canais de desvanecimento Hoyt, atualmente disponível na literatura em [7, Eq. 18] na forma de múltiplas integrais.

\section{B. Combinação por Ganho Igual}

Considerando a técnica EGC, a envoltória na saída do combinador $\tilde{R}$ pode ser escrita como

$$
\tilde{R}=\frac{\sum_{i=1}^{M} R_{i}}{\sqrt{M}}
$$

Neste caso, como antes, as formulações exatas para a LCR e AFD de canais Hoyt estão disponíveis na literatura na forma de múltiplas integrais [7, Eq. 14]. Sendo assim, usando as equações (11) e (5), aproximações altamente precisas para a LCR e AFD na saída do combinador serão apresentadas. Com o intuito de tornar estas expressões boas aproximações (isto é, $\tilde{R} \approx R$ ), nós calcularemos os parâmetros de $R(\Omega, \eta$ e $\mu$ ) a partir dos momentos exatos da saída do combinador $\tilde{R}$. Logo, segue que [3], [4], [5]

$$
\begin{gathered}
\Omega=E\left(\tilde{R}^{2}\right), \\
\eta_{a, b}=\frac{\sqrt{2 c}-\sqrt{3-2 c \pm \sqrt{9-8 c}}}{\sqrt{2 c}+\sqrt{3-2 c \pm \sqrt{9-8 c}}}, \\
c \triangleq \frac{\frac{E\left(\tilde{R}^{6}\right)}{\Omega^{3}}-\frac{3 E\left(\tilde{R}^{4}\right)}{\Omega^{2}}+2}{2\left[\frac{E\left(\tilde{R}^{4}\right)}{\Omega^{2}}-1\right]^{2}}, \\
\mu_{a, b}=\frac{\Omega^{2}}{E\left(\tilde{R}^{4}\right)-\Omega^{2}} \times \frac{1+\eta_{a, b}^{2}}{\left(1+\eta_{a, b}\right)^{2}} .
\end{gathered}
$$

Das equações acima, perceba que dois pares de estimadores para $\eta$ e $\mu$ são encontrados, chamados convenientemente de $\left(\eta_{a}, \mu_{a}\right)$ e $\left(\eta_{b}, \mu_{b}\right)$. O par correto será aquele que conduzir ao menor valor de $|E(\tilde{R})-E(R)|$, onde $E(R)$ é estimado de (4) para $(\Omega, \eta, \mu)=\left(\Omega, \eta_{a}, \mu_{a}\right)$ e $(\Omega, \eta, \mu)=\left(\Omega, \eta_{b}, \mu_{b}\right)$. Falta encontrarmos os momentos exatos $E\left(\tilde{R}^{k}\right)(k=1,2,4,6)$, como requeridos na formulação acima. Isso pode ser realizado a partir de (15) como

$$
\begin{array}{r}
E\left(\tilde{R}^{k}\right)=\frac{1}{M^{k / 2}} \sum_{k_{1}=0}^{k} \sum_{k_{2}=0}^{k_{1}} \ldots \sum_{k_{M-1}=0}^{k_{M-2}}\left(\begin{array}{c}
k \\
k_{1}
\end{array}\right)\left(\begin{array}{l}
k_{1} \\
k_{2}
\end{array}\right) \ldots\left(\begin{array}{l}
k_{M-2} \\
k_{M-1}
\end{array}\right) \\
E\left(R_{1}^{k-k_{1}}\right) E\left(R_{2}^{k_{1}-k_{2}}\right) \ldots E\left(R_{M}^{k_{M-1}}\right),
\end{array}
$$

onde $E\left(R_{i}^{j}\right)$ de cada termo Hoyt $R_{i}$ em (20) é obtido a partir de (4) com $R=R_{i}, \mu=0.5$ e $\eta=\left(1+b_{i}\right) /\left(1-b_{i}\right)$. Além disso, $\Omega_{i}=E\left(R_{i}^{2}\right)$.

\section{RESUltados NumÉRICOS}

Figs. 1 e 2 esboçam a LCR normalizada (eixo da esquerda), $N_{R}(r) / f_{m}$, e AFD (eixo da direita), $T_{R}(r) f_{m}$, como funções da envoltória normalizada $\rho=r / \hat{r}$. Aumentando $\mu$ e mantendo $\eta$ constante, perceba que níveis mais baixos são cruzados com taxas mais baixas, enquanto níveis mais altos são cruzados com taxas mais altas. Um comportamento similar é observado aumentando $\eta$ e mantendo $\mu$ constante. Comparando as Figs. 1 e 2, note que a variação do parâmetro $\mu$ tem uma influência maior no desempenho do sistema do que uma variação de $\eta$. As expressões analíticas para a LCR e AFD são validadas nas Figs. 3 e 4 usando simulação Monte Carlo. Tal simulação foi realizada gerando as variáveis autocorrelacionadas $X_{i}$ and $Y_{i}$ de acordo com o modelo de 


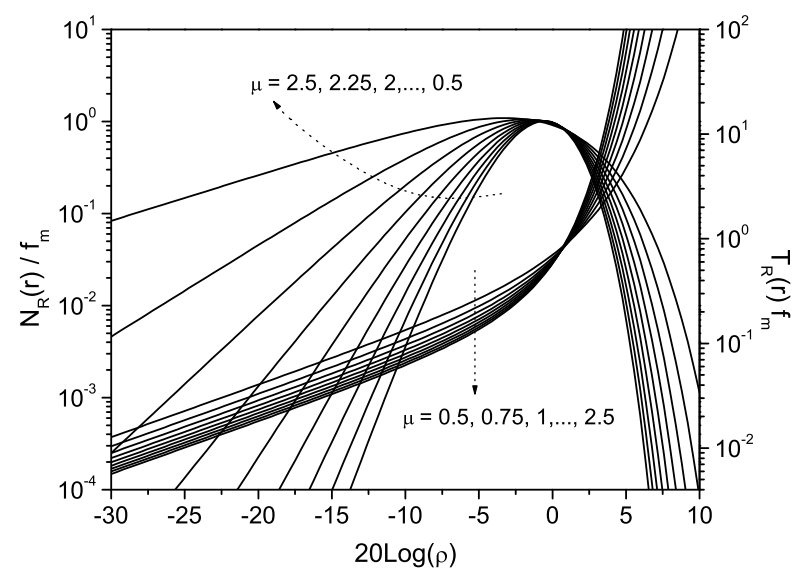

Fig. 1. LCR e AFD para canais de desvanecimento $\eta-\mu(\eta=0.5, \mu$ variando).

Jakes/Clark [17]. O parâmetro $\eta$ foi ajustado mudando a razão entre as potências de $X_{i}$ e $Y_{i}$ e o parâmetro $\mu$ foi introduzido variando o número de variáveis gaussianas como em (1).

A partir dos gráficos, observa-se uma excelente concordância entre as curvas teóricas e simuladas. Várias outras condições de desvanecimento foram investigadas e um excelente ajuste foi observado em todas elas. Fig. 5 plota as curvas teóricas e simuladas para a PCR de canais de desvanecimento $\eta-\mu$ e, mais uma vez, há um bom ajuste entre as curvas. Para $\mu=0.5$ (caso Hoyt), a PCR é independente do valor da fase, assumindo uma valor constante de $1 / 2 \sqrt{2}$, de acordo com [14].

Na Fig. 6, nossas aproximações para a LCR e AFD de sistemas EGC operando em canais de desvanecimento Hoyt são comparadas com as formulações exatas expressas em termos de múltiplas integrais como em [7, Eq. 14], para $M=2,3,4, b_{i}=0.1,0.6$ e $\Omega_{i}=1$. Perceba como as aproximações propostas produzem resultados indistingüíveis das formulações exatas. Como mencionado antes, para o caso MRC, nossas expressões alternativas, dadas em uma única integral, são de fato exatas e coincidem com as formulações em múltiplas integrais dadas em [7, Eq. 18]. É claro que, neste caso, não existe necessidade de comparar ambas as formulações.

\section{CONCLUSÕES}

Neste artigo, estatísticas de segunda ordem para canais de desvanecimento $\eta-\mu$ foram apresentadas. Dentre estas, as estatísticas conjuntas da envoltória, da fase e de suas derivadas temporais, assim como a PCR, foram obtidas em forma fechada. Por sua vez, a LCR e AFD foram expressas como uma única integral. Tais expressões foram validadas reduzindo-as a alguns casos particulares (Rayleigh, Hoyt e Nakagami-m), para os quais os resultados são conhecidos, e por meio de simulação. Além disso, baseando-se nos novos resultados, formulações exatas e na forma de uma única integral para a LCR e AFD de sistemas multiramos MRC

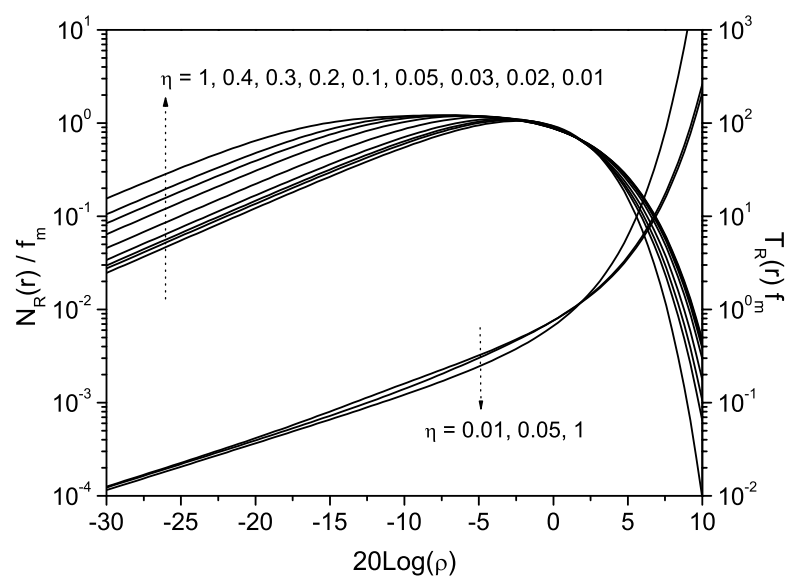

Fig. 2. LCR e AFD para canais de desvanecimento $\eta-\mu(\mu=0.6, \eta$ variando).

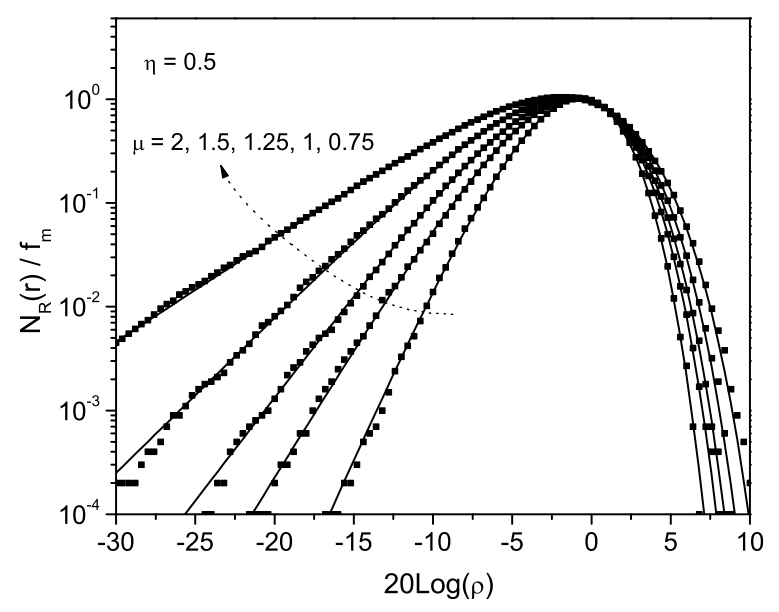

Fig. 3. Comparação entre as curvas analíticas e simuladas da LCR para canais de desvanecimento $\eta$ - $\mu$ (sólido $\rightarrow$ curvas analíticas; quadrado $\rightarrow$ curvas simuladas).

e aproximações altamente precisas para a LCR e AFD de sistemas multirramos EGC, operando em canais independentes de desvanecimento Hoyt, foram propostas.

\section{REFERÊNCIAS}

[1] Q. T. Zhang, A generic correlated Nakagami fading model for wireless communications, IEEE Trans. Commun., v. 51, $\mathrm{n}^{\circ} 11$, p. 1745-1748, Nov., 2003.

[2] G. D. Durgin, T. S. Rappaport, and D. A. de Wolf, New analytical models and probability density functions for fading in wireless communications, IEEE Trans. Commun., v. 50, n 6, p. 1005-1015, Jun., 2002.

[3] M. D. Yacoub, The $\eta-\mu$ distribution: A general fading distribution, IEEE Atlantic City Fall Veh. Tech. Conf., Boston, USA, Sep., 2000.

[4] M. D. Yacoub, The $\kappa-\mu$ and the $\eta$ - $\mu$ distribution, IEEE Ant. and Prop. Magazine, v. 49, $\mathrm{n}^{\circ}$ 1, p. 68-81, Feb., 2007.

[5] J. C. S. Santos Filho and M. D. Yacoub, Highly accurate $\eta-\mu$ approximation to the sum of $M$ independent non-identical Hoyt variates, IEEE Ant. and Wireless Prop. Lett., v. 4, p. 436-438, 2005. 


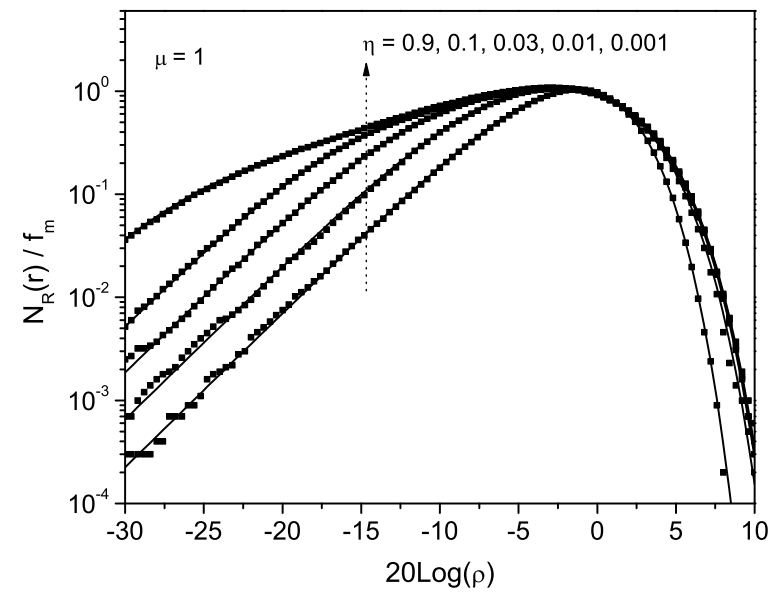

Fig. 4. Comparação entre as curvas analíticas e simuladas da LCR para canais de desvanecimento $\eta-\mu$ (sólido $\rightarrow$ curvas analíticas; quadrado $\rightarrow$ curvas simuladas).

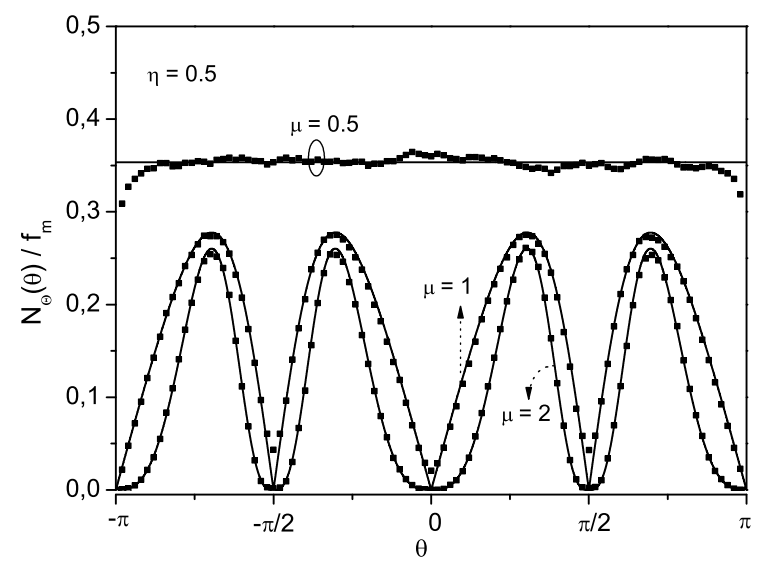

Fig. 5. Comparação entre as curvas analíticas e simuladas da PCR para canais de desvanecimento $\eta-\mu$ (sólido $\rightarrow$ curvas analíticas; quadrado $\rightarrow$ curvas simuladas).

[6] W. R. Braun and U. Dersch, A physical mobile radio channel model, IEEE Trans. Veh. Technol., v. 40, n 2, p. 472-482, May, 1991.

[7] G. Fraidenraich, J. C. S. Santos Filho, and M. D. Yacoub, Second-order statistics of maximal-ratio and equal-gain combining in Hoyt fading, IEEE Commun. Lett., v. 9, $\mathrm{n}^{\circ}$ 1, p. 19-21, Jan., 2005.

[8] M. Abramowitz and I. A. Stegun, Handbook of mathematical functions with formulas, graphs, and mathematical tables, New York: Dover, 1972.

[9] D. B. da Costa and M. D. Yacoub, The $\eta-\mu$ joint phase-envelope distribution, IEEE Ant. and Wireless Prop. Lett., vol. 6, p. 195-198, 2007.

[10] D. B. da Costa, M. D. Yacoub, J. C. S. Santos Filho, G. Fraindenraich, and J. R. Mendes, Generalized Nakagami-m phase crossing rate, IEEE Commun. Lett., v. 10, $\mathrm{n}^{\circ} 1$, p. 13-15, Jan., 2006.

[11] N. Youssef, C. -X. Wang, and M. Pätzold, A study on the second-order statistics of Nakagami-Hoyt mobile fading channels, IEEE Trans. Veh. Technol., v. 54, n 4, p. 1259-1265, Jul., 2005.

[12] M. D. Yacoub, G. Fraidenraich, and J. C. S. Santos Filho, Nakagami$\mathrm{m}$ phase-envelope joint distribution, Electron. Lett., v. 41, $\mathrm{n}^{\circ} 5$, Mar., 2005.

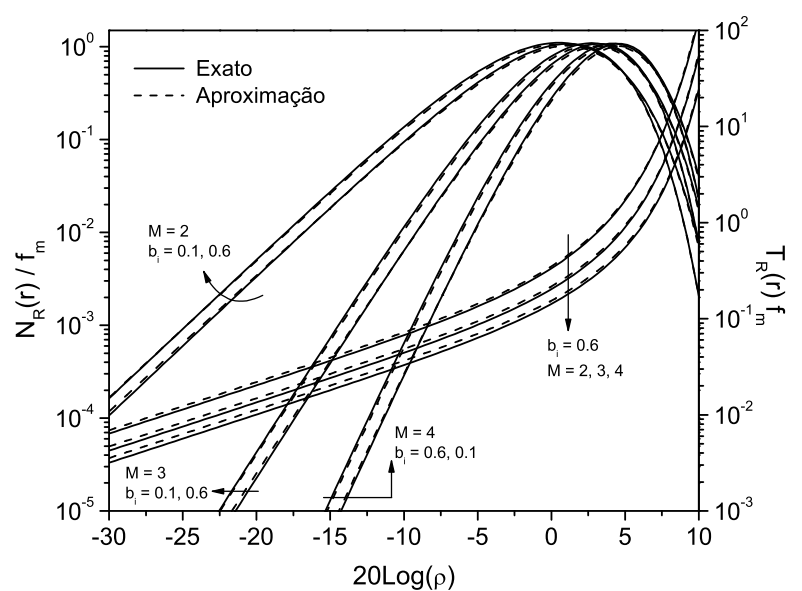

Fig. 6. Aproximação para a LCR e AFD de sistemas EGC multiramos operando em um ambiente de desvanecimento Hoyt pela LCR e AFD da envoltória $\eta-\mu$.

[13] M. D. Yacoub, J. E. V. Bautista, and L. G. R. Guedes, On high order statistics of the Nakagami-m distribution, IEEE Trans. Veh. Technol., v. 48, n 3, p. 790-793, May, 1999.

[14] N. Youssef, W. Elbahri, M. Pätzold, and S. Elasmi, On the crossing statistics of phase processes and random FM noise in Nakagami- $q$ mobile fading channels, IEEE Trans. on Wireless Commun., v. $4, \mathrm{n}^{\circ}$ 1, p. 24-29, Jan., 2005.

[15] M. D. Yacoub, C. R. C. M. da Silva, and J. E. V. Bautista, Secondorder statistics for diversity-combining techniques in Nakagami-fading channels, IEEE Trans. Veh. Technol., v. 50, ${ }^{\circ}$ 6, p. 1464-1470, Nov., 2001.

[16] C. -D. Iskander and P. T. Mathiopoulus, Analytical level crossing rates and average fade durations for diversity techniques in Nakagami fading channels, IEEE Trans. Commun., v. 50, $\mathrm{n}^{\circ}$ 8, p. 1301-1309, Aug., 2002.

[17] W. C. Jakes, Microwave Mobile Communications, New York: Wiley, 1974. 